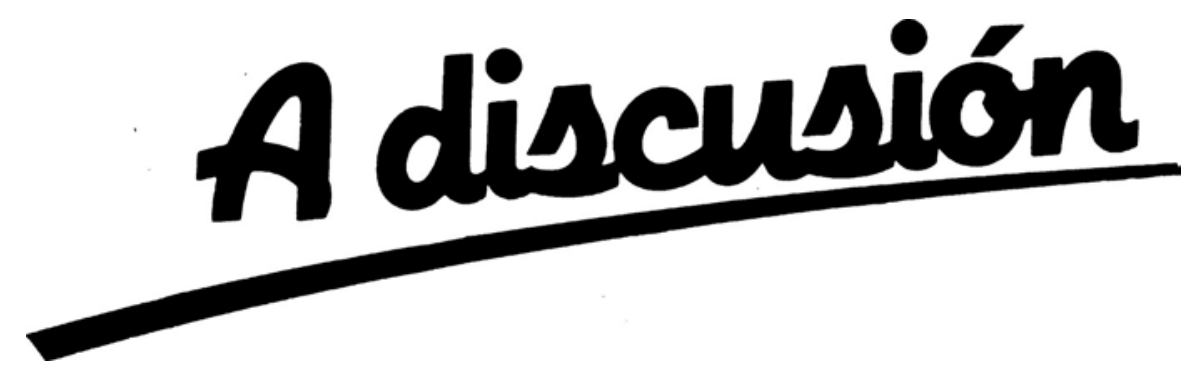

\title{
INCREASING RETURNS AND HETEROGENEITY IN A SPATIAL ECONOMY*
}

\section{Pascal Mossay}

WP-AD 2003-04

Correspondence to: Universidad de Alicante. Departamento de Fundamentos del Análisis Económico. Campus San Vicente del Raspeig, s/n. 03071 Alicante. E-mail: mossay@merlin.fae.ua.es.

Editor: Instituto Valenciano de Investigaciones Económicas, S.A.

Primera Edición Febrero 2003

Depósito Legal: V-895-2003

IVIE working papers offer in advance the results of economic research under way in order to encourage a discussion process before sending them to scientific journals for their final publication.

* I would like to thank Jacques Thisse for useful help and suggestions. The usual disclaimer applies. Financial support from the Commission of the European Communities, under grant HPMD-CT-200000010, is acknowledged. 


\title{
INGREASING RETURNS AND HETEROGENEITY \\ IN A SPATIAL ECONOMY
}

\section{Pascal Mossay}

\begin{abstract}
We study a general equilibrium model of global trade and local migration in a continuous geographical space. Trade is based on the Dixit-Stiglitz model of monopolistic competition. Migration is modelled as a local interaction decision process. Incentives for migration are of two types: homogeneous incentives of the group, associated with the identity of taste for higher utility levels, and heterogeneous incentives, due to idiosyncrasies in location taste. The impact of migration on the regional structure is twofold. First, when driven by utility differentials, it contributes to agglomeration because of the presence of increasing returns. Second, when reflecting heterogeneous individual choices, it fosters regional convergence. Furthermore, the size of agglomerations, when they occur, increases with the taste for variety and the proportion of the manufacturing population, and decreases with transport costs.
\end{abstract}

Keywords: heterogeneity, agglomeration, dispersion, monopolistic competition, trade, migration, normal modes

J.E.L. Classification: F12, R12, R23 


\section{Introduction}

The purpose of this paper is to develop a general equilibrium model combining both trade and migration in a continuous geographical space, and to show how increasing returns, transport costs and heterogeneity in location taste may affect the convergence process across locations.

Recent works by Krugman $(1991,1993,1996)$ have been particularly useful for understanding how increasing returns and labor mobility affect regional convergence. The basic framework is the following. There are two sectors: manufacturing and agriculture. Manufacturing employs mobile workers and agriculture immobile peasants. Consumers buy the manufacturing varieties on monopolistically competitive regional markets and the agricultural good on a competitive national market. Scale returns at the firm level contributes to regional divergence. This is because the more workers in a region, the more varieties in that region, and the higher their utility as they value variety. In turn this triggers additional inflows of workers in the region. On the other hand, the immobility of peasants contributes to regional convergence because firms locate close enough to the local markets they supply, so as to avoid prohibitive transport costs when supplying the immobile peasants in the unagglomerated areas.

In the case of a two-region model, conditions leading to convergence or divergence are related to the relative importance of increasing returns, transport costs and the labor proportion in the total population as shown by Krugman (1991). In a multi-location version of the same model, numerical simulations suggest that multiple agglomerations systematically emerge and are roughly evenly spaced across the landscape, see Krugman (1993). In a continuous location version of his model, Krugman (1996) showed that the economy always displays regional divergence. He also characterized the shape of the emerging agglomeration by performing numerical computations of the preferred wavelength. Similar results are obtained in the case of a large circular economy, see Fujita et al. (1999).

In this paper, trade modelling is built on the continuous models of Krugman (1996) and Fujita et al. (1999). Along a circular geographical space, there is a monopolistically competitive manufacturing sector employing mobile workers and a perfectly competitive agricultural sector employing immobile peasants. Flows of goods take place on the international scene. The Chamberlinian imperfect competitive economies are allowed to trade and product differentiation makes trade desirable. Specifically, product differen- 
tiation leads to gains from trade even when economies have identical consumption tastes, production technology, and factor endowments.

Our paper departs from Krugman's work in the migration modelling. In Krugman's work, higher utility level locations attract higher proportions of the total labor force. Location real wages provide an incentive for moving, and the worker's cost inhibates his response to real wage differentials. Many other factors contribute to the decision of a worker to migrate. Among them, personal circumstances (e.g. life-cycle considerations such as marriage or divorce) play an important role in the decision to migrate, see, e.g. Shaw (1975) or Greenwood (1985). Even though these personal circumstances may be quite diverse and therefore difficult to model in an explicit way, we may still want to identify the aggregate role these idiosyncrasies in location taste may have on regional convergence.

Migration will be supposed to occur locally. Workers are assumed to move to contiguous locations only. This reflects a local access to information. In this paper, there are two types of incentives to migrate: homogeneous incentives, associated with the identity of taste for higher utility levels, and heterogeneous incentives, due to idiosyncrasies in location taste. Under purely homogeneous incentives, workers move in the direction of the indirect utility gradient. These incentives are analogous to the ones used by Sonnenschein (1981, 1982) to model the convergence of a spatial competitive economy, where firms move across space in the direction of the profit gradient. On the other hand, idiosyncrasies in location taste will be assumed identically and independently distributed (iid) across workers and will correspond to a random walk in which worker's location is incremented stochastically. At the aggregate level this corresponds to a diffusion process, see Isard (1999). This approach is also largely used in population ecology to model behavioral or environmental stochasticity, see Murray (1993).

The impact of migration on the regional structure is twofold. First, when driven by utility differentials, it contributes to agglomeration because of the presence of increasing returns resulting from the taste for variety like in Krugman (1996) and Fujita et al. (1999). Second, when reflecting heterogeneous individual choices, it disperses labor across locations and therefore fosters regional convergence. Provided that heterogeneity in location taste is large enough, regional convergence may well happen. Our result thus differs qualitatively from Krugman (1996) and Fujita et al. (1999) in which instability always occurs. Furthermore, the size of agglomerations, when they occur, increases with the taste for variety and the proportion of the manufacturing 
population, and decreases with transport costs.

To study the convergence process in a continuous space, we need an adequate tool to analyze the spatial stability of the uniform long-run equilibrium. We will apply the normal mode method which is a standard linear spatial stability method in the hydrodynamic stability literature, see, e.g. Drazin and Reid (1991). The general idea is to find the conditions under which a small spatial perturbation is stable or not. To do so, the spatial perturbation is decomposed as a sum of elementary periodic perturbations. We then study whether each of the elementary periodic perturbation grows or is damped over time. If at least one of these elementary perturbations grows over time, then the long-run equilibrium is unstable. This technique has been applied by Krugman and Venables (1996) to study a spatial model of international specialization, and in Krugman (1996) and Fujita et al. (1999) to perform numerical computations of the preferred wavelength of emerging agglomerations, that is the wave-length of the dominant unstable perturbation. The corresponding discrete technique has also been used by Papageorgiou and Smith (1983). Their purpose was to find the conditions under which a spatial externality may lead the spatial uniform equilibrium to be unstable. We shall use the normal mode method to determine the conditions under which agglomerations may happen from local instability of a uniform long run equilibrium.

Section 2 lays out the spatial economy framework. The short-run equilibrium is described in section 3. We model migration as a local interaction process in section 4 . The long-run equilibrium is then defined in section 5. In section 6, in Proposition 1, we determine the conditions under which agglomerations can arise by performing a linear spatial stability analysis of the long-run equilibrium. Furthermore, we characterize the size of the emerging agglomerations in Proposition 2. Finally, section 7 summarizes the main results.

\section{The Economy}

The economy extends along a circle $\mathbb{C}$ of radius $R$ and is inhabited by a continuum of consumers (workers/peasants). There are two sectors in the economy: the manufacturing sector, which exhibits increasing returns to scale, and agriculture, which has constant returns.

Each consumer at location $x$ and time $t$ enjoys a Cobb-Douglas utility 
from the two types of goods

$$
C_{M}^{\mu}(x, t) C_{A}^{1-\mu}(x, t)
$$

where $\mu$ is the share of manufactured goods in expenditure, $C_{A}$ the consumption of the agricultural good, and $C_{M}$ the consumption of the manufactured aggregate which is defined by

$$
C_{M}(x, t)=\left[\int_{x-\pi R}^{x+\pi R}\left(\int_{0}^{n(z, t)} c_{i}(z, x, t)^{\frac{\sigma-1}{\sigma}} d i\right) d z\right]^{\frac{\sigma}{\sigma-1}}
$$

where $n(z, t)$ is the density of manufactured varieties available at location $z$, $c_{i}(z, x, t)$ is the consumption of variety $i$ produced at $z$, and $\sigma>1$ is the elasticity of substitution among manufactured varieties.

Farming is an activity that takes place under constant returns to scale

$$
A(x, t)=Q_{A}(x, t)
$$

where $A(x, t)$ is the density of peasants needed in $x$ at $t$ to produce $Q_{A}(x, t)$ units of the agricultural good.

Manufacturing variety $i$ involves a fixed cost and a constant marginal cost. Economies of scale are thus realized at the firm level

$$
L_{i}(x, t)=\alpha+\beta Q_{M, i}(x, t)
$$

where $L_{i}(x, t)$ is the amount of labor used in $x$ at $t$ to produce $Q_{M, i}(x, t)$ units of variety $i$.

Transport costs only affect manufactured goods and take the Samuelson iceberg form. More precisely, when the amount $Z$ of some variety is shipped from locations $z$ to $x$, then the amount $X$ of that variety which is effectively available at location $x$ is given by

$$
X=Z \exp [-\tau|x-z|]
$$

where $\tau$ is the transport cost per unit of distance, and $|x-z|$ the distance between locations $z$ and $x$.

We assume that there is a continuum of manufacturing firms. Each of them produces a single variety, and faces a demand curve with a constant elasticity $\sigma$; this will be confirmed below in equation (11). The optimal 
pricing behavior of any firm at location $x$ and time $t$ is therefore to set the price $p_{i}(x, t)$ of variety $i$ at a fixed markup over marginal cost

$$
p_{i}(x, t)=\frac{\sigma}{\sigma-1} \beta W(x, t)
$$

where $W(x, t)$ is the worker wage rate prevailing at location $x$.

Firms are free to enter into the manufacturing sector so that their profits are driven to zero. Consequently, their output is given by

$$
Q_{M, i}(x, t)=\frac{\alpha}{\beta}(\sigma-1)
$$

Since all varieties are produced at the same scale, the density $n(x, t)$ of manufactured goods produced at each location is proportional to the density $L(x, t)$ of workers at that location,

$$
L(x, t)=\int_{0}^{n(x, t)} L_{i}(x, t) d i=\alpha \sigma n(x, t)
$$

This relationship is crucial. When some workers move to a new location, they no longer produce the same mix of products but other differentiated products. As a result, varieties produced in one location are different from those produced in any other location. Since consumers are characterized by a preference for variety, they will buy from all locations so that trade of varieties between any location pair will arise. Hence trade is global.

\section{Short-Run Equilibrium}

Total income $Y$ at location $x$ and time $t$ is given by

$$
Y(x, t)=A p^{A}+L(x, t) W(x, t)
$$

where $A$ is the constant density of peasants, $L(x, t)$ the density of workers, and $p^{A}$ the price of the agricultural good.

Workers are not interested in nominal wages but rather in utility levels. In order to consume at $x$, one unit of variety $i$ produced at location $z, \exp [\tau|x-z|]$ units must be shipped so that the delivery price is $p_{i}(z, t)$ $\exp [\tau|x-z|]$. The price index of the manufactured aggregate for consumers at location $x, \Theta(x, t)$, is obtained by computing the minimum cost of purchasing one unit of the manufactured aggregate $C_{M}(x, t)$ 


$$
\Theta(x, t)=\left[\int_{x-\Pi R}^{x+\Pi R} \int_{0}^{n(z, t)} p_{i}(z, t)^{-(\sigma-1)} \exp [-\tau(\sigma-1)|z-x|] d z\right]^{-\frac{1}{(\sigma-1)}}
$$

By using the pricing rule (6) and relation $(8), \Theta(x, t)$ may be rewritten as

$$
\begin{aligned}
\Theta(x, t)= & \frac{\beta \sigma}{\sigma-1}(\alpha \sigma)^{1 /(\sigma-1)} \\
& \cdot\left[\int_{x-\Pi R}^{x+\Pi R} L(z, t) W(z, t)^{-(\sigma-1)} \exp [-\tau(\sigma-1)|z-x|] d z\right]^{-\frac{1}{(\sigma-1)}}(10)
\end{aligned}
$$

The consumption of variety $i \in[0, n(z, t)]$ produced at $z$ may be expressed for workers and peasants located at $x$ as follows

$$
\begin{aligned}
c_{i}^{w}(z, x, t) & =\mu W(x, t) p_{i}(z, t)^{-\sigma} \exp [-\tau(\sigma-1)|z-x|] \Theta(x, t)^{\sigma-1} \\
c_{i}^{a}(z, x, t) & =\mu p^{A} p_{i}(z, t)^{-\sigma} \exp [-\tau(\sigma-1)|z-x|] \Theta(x, t)^{\sigma-1}
\end{aligned}
$$

The total demand for variety $i$ produced at $z$ is obtained by integrating the demand for that variety of all the consumers along the circle $\mathbb{C}$,

$$
\begin{aligned}
Q_{M, i}^{D}(z, t)= & \int_{z-\Pi R}^{z+\Pi R}\left[L(x, t) c_{i}^{w}(z, x, t)+A c_{i}^{a}(z, x, t)\right] d x \\
= & \int_{z-\Pi R}^{z+\Pi R} \mu\left(L(x, t) W(x, t)+A p^{A}\right) p_{i}(z, t)^{-\sigma} \\
& \cdot \exp [-\tau(\sigma-1)|z-x|] \Theta(x, t)^{\sigma-1} d x
\end{aligned}
$$

By using the total income expression (9), we get

$$
Q_{M, i}^{D}(z, t)=\int_{z-\Pi R}^{z+\Pi R} \mu Y(x, t) p_{i}(z, t)^{-\sigma} \exp \left[-\tau(\sigma-1)|z-x| \Theta(x, t)^{\sigma-1} d x\right.
$$

The market-clearing condition for variety $i$ produced at $z$ is obtained by equating the demand $Q_{M, i}^{D}(11)$ and the supply $Q_{M, i}(z, t)(7)$ of that variety,

$$
p_{i}(z, t)=\left[\mu \frac{\beta}{\alpha(\sigma-1)} \int_{z-\Pi R}^{z+\Pi R} Y(x, t) \Theta(x, t)^{\sigma-1} \exp [-\tau(\sigma-1)|z-x|] d x\right]^{1 / \sigma}
$$


Because of the pricing rule (6), we get

$$
\begin{aligned}
W(z, t)= & \frac{\sigma-1}{\beta \sigma}\left[\frac{\mu \beta}{\alpha(\sigma-1)}\right]^{1 / \sigma} \\
& \cdot\left[\int_{z-\Pi R}^{z+\Pi R} Y(x, t) \Theta(x, t)^{\sigma-1} \exp [-\tau(\sigma-1)|z-x|] d x\right]^{1 / \sigma}
\end{aligned}
$$

The manufacturing wage $W(z, t)$ is the wage prevailing at location $z$ and time $t$ such that firms at $z$ break even given the income levels $Y(x, t)$, the price indices $\Theta(x, t)$ in all locations, and the transportation cost technology.

The indirect utility $\Omega(x, t)$ of a worker located at $x$ is then obtained through (1) by

$$
\begin{aligned}
\Omega(x, t) & =U(\Theta(x, t), W(x, t)) \\
& =C_{M}^{\mu}(\Theta(x, t), W(x, t)) C_{A}^{1-\mu}(\Theta(x, t), W(x, t)) \\
& =(\mu W(x, t) / \Theta(x, t))^{\mu}\left[(1-\mu) W(x, t) / p^{A}\right]^{1-\mu} \\
& =\mu^{\mu}(1-\mu)^{1-\mu}\left(p^{A}\right)^{-(1-\mu)} \Theta^{-\mu}(x, t) W(x, t)
\end{aligned}
$$

Definition 1 A short-run equilibrium at location $x$ and time $t$, is defined, taking $L(x, t)$ as given, by equations (9), (10), (12), (13).

\section{Migration Behavior}

We now introduce dynamics by specifying a local process of reallocation of the labor force across locations. We first describe the worker migration decision; we then aggregate individual decisions to obtain the equation governing the evolution of labor over space and time.

We want to capture the idea that each worker faces an arbitrage when deciding to migrate. The trade-off results from the common incentive he finds in higher utility levels that he can earn in neighboring locations and idiosyncratic incentives due to his own individual preference for local places.

Each worker has a preference relation which is split into two components. The first component is defined over the observable consumption $C_{A}, C_{M}$ at a given location. Workers are assumed to be homogeneous with respect to consumption, meaning that all workers at location $x$ make the same con-

sumption decisions. The second component is defined over individual tastes 
for local places: a worker in location $x$ may want to migrate according to the indirect utility gradient, or to move left, right, or stay put, irrespective of the utility gradient. As individual motives to migrate may be very diverse, it seems natural to recognize that they are not known to the modeller. By contrast, we assume that the modeler knows the distribution of migration due to the heterogeneity of workers in their preference for local places; see Anderson et al. (1992). This means that the modeler is unable to exactly predict the worker's migration decision, and can at best predict the probability that a worker will make a given migration choice. In the approach used here, uncertainty is due to the lack of information available to the modeler. However, this approach can be seen as equivalent to a random utility approach where the random variable would reflect stochastic migration taste differences among workers, see Anderson et al. (1992). For this reason, the approach followed here differs substantially from the one use in deterministic models dealing with tastes for location, such as, for instance, Greenberg (1983), or Mansoorian and Myers (1997).

At the aggregate level the effect of the heterogeneity in location taste on the spatial distribution of labor is to contribute to the dispersion of labor across locations. Diffusion processes such as suggested in Isard (1999) can thus be interpreted as resulting from the aggregation of idiosyncrasies in location taste. This approach is also largely used in population ecology to model behavioral or environmental stochasticity, see Murray (1993). We will assume that idiosyncratic migration taste differences do not depend on the direction of migration. Otherwise there would be either absolute east- or west- lover migrant, in which case trivial agglomeration to the east or to the west would arise. Furthermore, the probabilities of moving are supposed to be the same for all workers. This will allow us to describe the average behavior of labor, by getting an evolution law for the average density of workers.

We model the workers' migration process as follows. Labor population is distributed along the circle $\mathbb{C}$. At each point in time $t$, a worker at location $x$ faces the indirect utility gradient $\partial \Omega / \partial x$. He responds to it with probability $1-\gamma$, by moving in that direction. If not, he decides, as a result of his individual taste, to move left, right, or stay at $x$ with equal probabilities, that is $\gamma / 3$; see Figure 1.

If the adjustment speed to utility differentials is captured by $\xi$, when deciding to move in the utility gradient direction, workers move over the distance $V^{\prime} d t$ during time span $d t$ with 


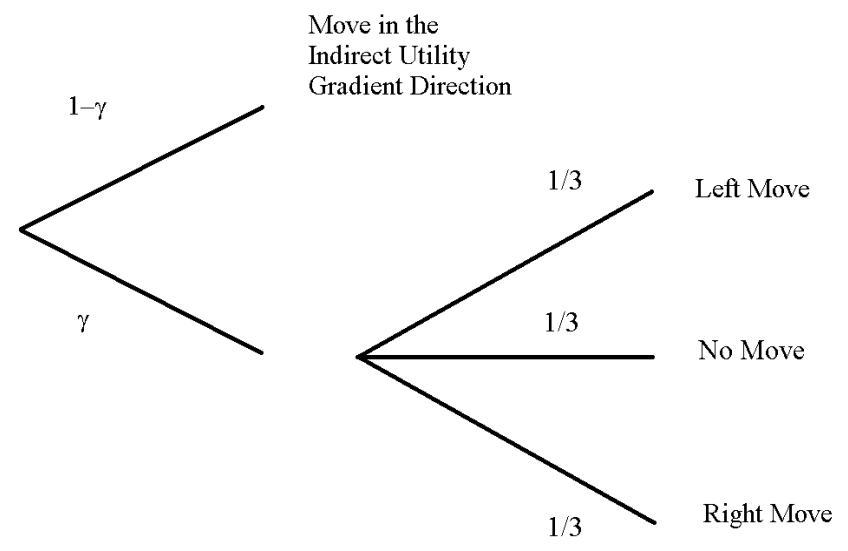

Figure 1: Choice Probabilities of Moving

$$
V^{\prime}=\xi \frac{\partial \Omega}{\partial x}
$$

On the other hand, migration due to the heterogeneity in taste for local places is modeled as the following brownian motion. When migration is not motivated by utility differentials, a worker located at $x$ moves to $x+d \lambda$, $x-d \lambda$ or stays at $x$ with equal probabilities during time span $d t$.

This implies that migration is viewed as a local process, in the sense that no straight migration takes place between two locations separated by a given, finite distance. This is consistent with a local informational setting. Nevertheless over time, the continuous flows of migrants do not preclude finite, or even large scale migration between any two locations. The local migration behavior is consistent with empirical findings according to which the intensity of migration flows declines with the increasing distance between origin and destination, see, for instance, Shaw (1975).

Consider a worker in location $x$ at time $t$. The probability for that worker to be at location $x$ (meaning a location between $x$ and $x+d x$ ) is denoted $q(x, t) d x$. We now describe the location a worker may be coming from. From 
an inductive inspection of Figure 1, it is clear that a worker in location $x$ at time $t$ may be coming from four different locations: (i) $x-V^{\prime} d t$, (ii) $x-d \lambda$, (iii) $x+d \lambda$, or (iv) $x$. To establish the probability law, that is how the density $q(x, t)$ evolves over time and space, we assume for expositional simplicity that the velocity $V^{\prime}$ of the worker is constant (see Appendix 1 for the case where $V^{\prime}$ may vary over space and time). At time $t-d t$, the worker was in $x-V^{\prime} d t$ with probability $q\left(x-V^{\prime} d t, t-d t\right) d x$ and chose to move to $x$ with probability $(1-\gamma)$ as a result of the common incentive for higher utility levels, see Figure 1. Also, he was in $x-d \lambda, x+d \lambda$, or $x$ with probabilities $q(x-d \lambda, t-d t) d x, q(x+d \lambda, t-d t) d x, q(x, t-d t) d x$ and chose to move to $x$ with probability $\gamma / 3$ as a result of the heterogeneity in taste for local places, see Figure 1. It then follows that the probability $q(x, t)$, at time $t$, for the worker to be at location between $x$ and $x+d x$ is given by

$$
\begin{aligned}
q(x, t) d x= & \gamma\left[\frac{1}{3} q(x-d \lambda, t-d t)+\frac{1}{3} q(x+d \lambda, t-d t)+\frac{1}{3} q(x, t-d t)\right] d x \\
& +(1-\gamma) q\left(x-V^{\prime} d t, t-d t\right) d x
\end{aligned}
$$

So as to establish the probability law, we take the Taylor expansion of the RHS. This yields

$$
\begin{aligned}
q(x-d \lambda, t-d t)= & q(x, t)-d \lambda \frac{\partial q}{\partial x}-d t \frac{\partial q}{\partial t}+\frac{1}{2}(d \lambda)^{2} \frac{\partial^{2} q}{\partial x^{2}} \\
& +\frac{1}{2} d t^{2} \frac{\partial^{2} q}{\partial t^{2}}+\frac{1}{2} d \lambda d t \frac{\partial^{2} q}{\partial x \partial t}+\ldots \\
q(x+d \lambda, t-d t)= & q(x, t)+d \lambda \frac{\partial q}{\partial x}-d t \frac{\partial q}{\partial t}+\frac{1}{2} d \lambda^{2} \frac{\partial^{2} q}{\partial x^{2}} \\
& +\frac{1}{2} d t^{2} \frac{\partial^{2} q}{\partial t^{2}}-\frac{1}{2} d \lambda d t \frac{\partial^{2} q}{\partial x \partial t}+\ldots \\
q\left(x-V^{\prime} d t, t-d t\right)= & q(x, t)-V^{\prime} d t \frac{\partial q}{\partial x}-d t \frac{\partial q}{\partial t}+\frac{1}{2}\left(V^{\prime} d t\right)^{2} \frac{\partial^{2} q}{\partial x^{2}} \\
& +\frac{1}{2} d t^{2} \frac{\partial^{2} q}{\partial t^{2}}+\frac{1}{2} V^{\prime} d t^{2} \frac{\partial^{2} q}{\partial x \partial t}+\ldots \\
q(x, t-d t)= & q(x, t)-d t \frac{\partial q}{\partial t}+\frac{1}{2} d t^{2} \frac{\partial^{2} q}{\partial t^{2}}+\ldots
\end{aligned}
$$

Substituting (16) in (15) and assuming that $d \lambda^{2} / d t$ is finite ${ }^{1}$, we obtain the

$1 \frac{d \lambda^{2}}{d t}$ remains finite as $d t \rightarrow 0, d \lambda \rightarrow 0$. 
probability evolution law governing $q(x, t)$

$$
\frac{\partial q}{\partial t}(x, t)+\frac{\partial}{\partial x}(V(x, t) q(x, t))=\frac{\partial}{\partial x}\left(d \frac{\partial q}{\partial x}(x, t)\right)
$$

where $V$ and $d$ are defined repectively by $(1-\gamma) V^{\prime}$ and $\gamma / 3(d \lambda)^{2} / d t$. This means that $d$ measures the heterogeneity in location taste. In Appendix 1 , it is shown that equation (17) is still valid when $V^{\prime}(x, t)$ varies with the intensity of the utility gradient (14). Then $V(x, t)$ is given by

$$
V(x, t)=(1-\gamma) V^{\prime}(x, t)=(1-\gamma) \xi \frac{\partial \Omega}{\partial x}(x, t)=m \frac{\partial \Omega}{\partial x}(x, t)
$$

where $m$ defined by $(1-\gamma) \xi$ measures the identity of taste for higher utility levels.

We now describe the labor population by its average behavior. The description says how many workers end up moving there and consuming what, but does not describe individual behavior per se. The evolution law for the average density of workers $L(x, t)$ is then obtained by multiplying the equation (17) by the total number of workers $\widetilde{L}$ in the economy

$$
\frac{\partial L}{\partial t}(x, t)+\frac{\partial}{\partial x}(V(x, t) L(x, t))=\frac{\partial}{\partial x}\left(d \frac{\partial L}{\partial x}(x, t)\right)
$$

where $L(x, t)$ corresponds to $q(x, t) \widetilde{L}$ while $V$ is given by (18).

\section{Interpretation of the evolution equation}

Equation (19) may be rewritten as

$$
\frac{\partial L}{\partial t}(x, t)=-\frac{\partial}{\partial x} \Phi(x, t)
$$

where the labor flow $\Phi$ may be decomposed as

$$
\Phi(x, t)=\Phi_{v}(x, t)+\Phi_{d}(x, t)
$$

with $\Phi_{v}=V L$ and $\Phi_{d}=-d \partial L / d x$.

Labor flow $(\Phi)$ results from a utility differential effect (through $\Phi_{v}$ ) and from idiosyncrasies in location taste (through $\Phi_{d}$ ). Let $\Gamma=\left[x_{1}, x_{2}\right]$ be a region, and denote $\int_{x_{1}}^{x_{2}} L(x, t) d x$ the labor population in $\Gamma$ at time $t$ by $\mathcal{L}(t)$. By integrating (20) over region $\Gamma$, we get

$$
\frac{d}{d t} \mathcal{L}(t)=-\left[\Phi\left(x_{2}, t\right)-\Phi\left(x_{1}, t\right)\right]
$$




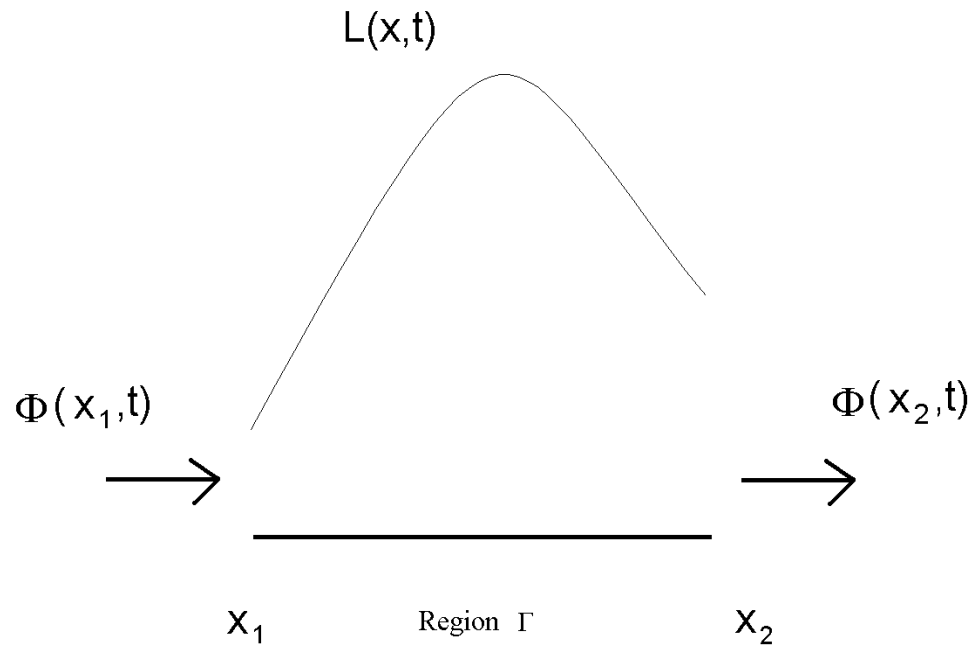

Figure 2: Conservation Law of Labor

Equation (22) is a conservation law of the labor population. It asserts that the rate of increase of the labor population $\mathcal{L}(t)$ in region $\Gamma$, is equal to the total labor flow $\Phi(x, t)$ into $\Gamma$ across its borders $x_{1}$ and $x_{2}$; see Figure 2. Therefore, equation (20) is called the conservative form corresponding to (22).

When there is no heterogeneity among workers (i.e., $\Phi_{d}=\gamma=0$ ), the net increase of workers in region $\Gamma$ during time span $[t, t+d t]$ is due only to the attraction of workers because of higher utility levels

$$
\begin{aligned}
\mathcal{L}(t+d t)-\mathcal{L}(t) & \simeq\left[\Phi_{v}\left(x_{1}, t\right)-\Phi_{v}\left(x_{2}, t\right)\right] d t \\
& =\left(V\left(x_{1}, t\right) L\left(x_{1}, t\right)-V\left(x_{2}, t\right) L\left(x_{2}, t\right)\right) d t
\end{aligned}
$$

On the other hand, when there is no systematic migration in the direction of the utility gradient (i.e., $\Phi_{v}=1-\gamma=0$ ), the net increase of workers in region $\Gamma$ during time span $[t, t+d t]$ is due to the dispersion of workers as a consequence of their heterogeneity

$$
\begin{aligned}
\mathcal{L}(t+d t)-\mathcal{L}(t) & \simeq\left[\Phi_{d}\left(x_{1}, t\right)-\Phi_{d}\left(x_{2}, t\right)\right] d t \\
& =d\left[-\frac{\partial L}{\partial x}\left(x_{1}, t\right)+\frac{\partial L}{\partial x}\left(x_{2}, t\right)\right] d t
\end{aligned}
$$




\section{Long-Run Equilibrium Analysis}

An adequate change of variables (see Appendix 2) allows us to write (9), (10), (12), (13), (18), and (19) in the following way

$$
\begin{gathered}
Y(x, t)=(1-\mu)+\mu L(x, t) W(x, t) \\
\Theta(x, t)=\left[\frac{1}{C} \int_{(x-\Pi R) \tau(\sigma-1)}^{(x+\Pi R) \tau(\sigma-1)} L(z, t) W(z, t)^{-(\sigma-1)} \exp [-|z-x|] d z\right]^{-\frac{1}{(\sigma-1)}} \\
W(x, t)=\left[\frac{1}{C} \int_{(x-\Pi R) \tau(\sigma-1)}^{(x+\Pi R) \tau(\sigma-1)} Y(z, t) \Theta(z, t)^{\sigma-1} \exp [-|z-x|] d z\right]^{1 / \sigma} \\
\Omega(x, t)=\Theta(x, t)^{-\mu} W(x, t) \\
\frac{\partial L}{\partial t}(x, t)+\frac{\partial}{\partial x}(V(x, t) L(x, t))=\Delta \frac{\partial^{2} L}{\partial x^{2}}(x, t) \\
V(x, t)=\frac{\partial \Omega}{\partial x}(x, t)
\end{gathered}
$$

where $C$ is defined by $2[1-\exp (-\Pi R \tau(\sigma-1))]$ and $\Delta$ is a mobility index measuring the relative importance of two effects: the heterogeneity in location taste and the identity of taste for higher utility levels, see Appendix 2. When the heterogeneity in location taste among consumers is high, then the mobility index $\Delta$ is high. On the other hand, when the drift towards higher utility levels is the dominant spatial force, then the mobility index $\Delta$ is low.

The dynamics of the model is governed by equations (27), (28), (29), (30), (31), and (32). This set of equations describes the spatial and temporal evolution of the state variables.

Definition 2 A long-run equilibrium is defined as a stationary state of the system of equations (27), (28), (29), (30), (31), and (32). 
Such a long-run equilibrium is the uniform stationary state defined by

$$
\begin{aligned}
\bar{Y} & =(1-\mu)+\mu \bar{L} \bar{W} \\
\bar{\Theta} & =\left[\frac{\bar{L} \bar{W}^{-(\sigma-1)}}{C} \int_{-\Pi R \tau(\sigma-1)}^{+\Pi R \tau(\sigma-1)} \exp [-|z|] d z\right]^{-\frac{1}{(\sigma-1)}} \\
\bar{W} & =\left[\frac{\bar{Y} \bar{\Theta}^{\sigma-1}}{C} \int_{-\Pi R \tau(\sigma-1)}^{+\Pi R \tau(\sigma-1)} \exp [-|z|] d z\right]^{\frac{1}{\sigma}} \\
\bar{\Omega} & =\bar{W}^{-\mu} \\
\bar{V} & =0
\end{aligned}
$$

When $\bar{L}=1$, the uniform steady state is defined by

$$
\bar{L}=1 ; \bar{Y}=1 ; \bar{\Theta}=1 ; \bar{W}=1 ; \bar{\Omega}=1 ; \bar{V}=0
$$

In such an equilibrium, the net flow of workers through any location is zero.

From now on, we will be concerned with the long-run equilibrium (33). To address the regional convergence issue, we will study the dynamics of the system around this long-run equilibrium.

\section{Agglomeration as Local Instability}

In this section we analyze the spatial stability of the long-run equilibrium (33). The idea is to find the conditions under which a small spatial pertubation is stable or not. We will restrict our attention to the perturbations which are in some sense close to the long-run equilibrium (33). This allows us to focus on the linearized equations of the system. In order to study the time evolution of a spatial perturbation, we decompose it as a sum of elementary periodic perturbations. The reason for doing so is that any arbitrary perturbation may be expressed as a linear combination of periodic perturbations according to the Fourier decomposition. For the sake of simplicity, periodic perturbations may be viewed as $\sin (k x)$. High (low) values of $k$ correspond to high (low) frequency perturbations. Later on, we need to introduce more general periodic perturbations called normal modes. We then study whether each of these elementary periodic perturbations grows or is damped over time. If at least one of the elementary periodic perturbations is unstable, that is growing over time, then the long-run equilibrium is unstable. More 
details concerning the normal mode stability analysis may be found in a general reference in the hydrodynamic stability literature, see, e.g. Drazin and Reid (1991).

\subsection{Perturbation Linearized Equations}

In order to perform the linearization of equations (27), (28), (29), (30), (31), and (32), we decompose the variables into their steady state value and their corresponding deviation. We denote these deviations by $y, \theta, w, \omega, l, v$, and write

$$
\begin{aligned}
& L(x, t)=1+l(x, t) ; Y(x, t)=1+y(x, t) ; W(x, t)=1+w(x, t) \\
& \Theta(x, t)=1+\theta(x, t) ; \Omega(x, t)=1+\omega(x, t) ; V(x, t)=0+v(x, t)
\end{aligned}
$$

The perturbation equations are then obtained by the substitution of (34) in (27), (28), (29), (30), (31), and (32). Neglecting second-order terms such as $l w$ leads to the following perturbation linearized equations (see Appendix 3)

$$
\begin{aligned}
y(x, t) & =\mu(l(x, t)+w(x, t)) \\
\theta(x, t) & =\frac{1}{C} \int_{(x-\Pi R) \tau(\sigma-1)}^{(x+\Pi R) \tau(\sigma-1)}\left(-\frac{1}{(\sigma-1)} l(z, t)+w(z, t)\right) \exp [-|x-z|] d z \\
w(x, t) & =\frac{1}{\sigma C} \int_{(x-\Pi R) \tau(\sigma-1)}^{(x+\Pi R) \tau(\sigma-1)}(y(z, t)+(\sigma-1) \theta(z, t)) \exp [-|x-z|] d z \\
\omega(x, t) & =w(x, t)-\mu \theta(x, t) \\
\frac{\partial l}{\partial t}(x, t)+\frac{\partial v}{\partial x}(x, t) & =\Delta \frac{\partial^{2} l}{\partial x^{2}}(x, t) \\
v(x, t) & =\frac{\partial \omega}{\partial x}(x, t)
\end{aligned}
$$

\subsection{Normal Mode Method}

The main idea in what follows is to study how elementary periodic perturbations evolve over time. To deal with our problem, we introduce the following spatial normal modes.

Definition 3 A spatial normal mode is determined by its frequency $k$, and is defined as $\exp [I k x]$, with $I^{2}=-1$. 

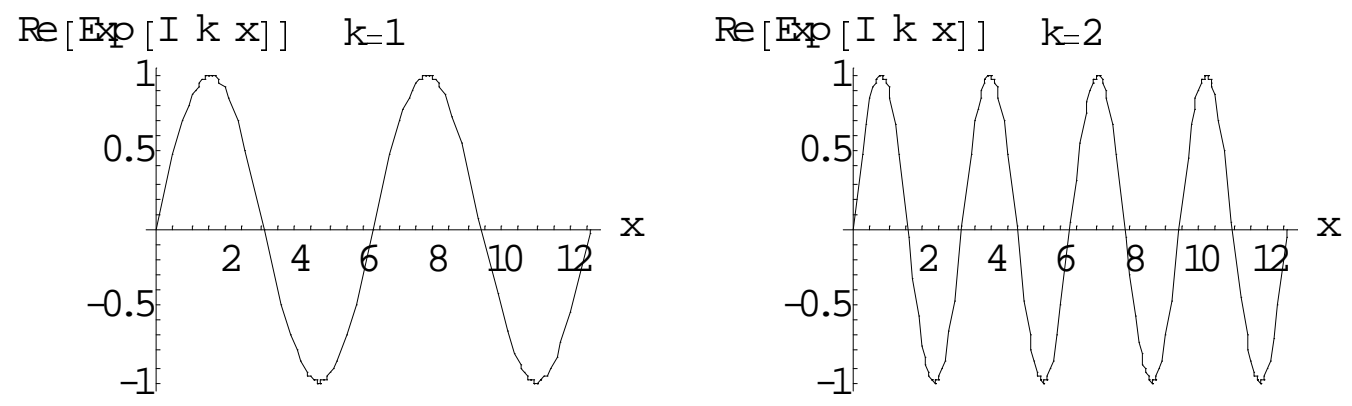

Figure 3: Representation of Spatial Modes $(k=1, k=2)$

As suggested in the case of sinusoidal functions, high (low) frequency spatial modes have a low (large) spatial scale. The sinusoidal variations correspond to a succession of alternating rich and poor areas. While large spatial scales refer to configurations consisting in a small number of large areas, low spatial scales refer to configurations consisting in a large number of small areas. This periodic organization of the economic activities across locations reminds the location theory of central places. Spatial theorists like Christaller (1966) or Lösch (1954) have addressed the shape that market areas should optimally display. According to their works, it turns out that very regular symmetric patterns arise in a spatial economy. The optimal shape of market areas is the hexagone: it maximizes the welfare per unit of space. The symmetric and regular nature of spatial modes is depicted in Figure 3. The spatial scale of the mode $k=2$ is lower than the spatial scale of the mode $k=1$, see Figure 3. This is because the alternation of poor and rich areas takes place over a smaller distance in the case $k=2$.

Since equations (35) are linear, this suggests that one looks for solutions where all perturbations are proportional to $\exp [s t+I k x]$, with $I^{2}=-1$, and where $s(k)$ denotes the amplification factor related to spatial mode $k$. So 
perturbations are assumed to be of the following type

$$
\left[\begin{array}{l}
y(x, t) \\
\theta(x, t) \\
w(x, t) \\
\omega(x, t) \\
l(x, t) \\
v(x, t)
\end{array}\right]=\exp [s t+I k x]\left[\begin{array}{l}
y_{0} \\
\theta_{0} \\
w_{0} \\
\omega_{0} \\
l_{0} \\
v_{0}
\end{array}\right]
$$

where $y_{0}$ is the constant amplitude of the total income perturbation density; and similarly for $\theta_{0}, w_{0}, \omega_{0}, l_{0}$, and $v_{0}$. Substituting (36) in (35) yields

$$
\begin{aligned}
y_{0} & =\mu\left(l_{0}+w_{0}\right) \\
\theta_{0} & =-\frac{1}{\sigma-1} h l_{0}+h w_{0} \\
w_{0} & =\frac{1}{\sigma} h y_{0}+\frac{\sigma-1}{\sigma} h \theta_{0} \\
\omega_{0} & =w_{0}-\mu \theta_{0} \\
s l_{0}+I k v_{0} & =-\Delta k^{2} l_{0} \\
v_{0} & =I k \omega_{0}
\end{aligned}
$$

where $h$ is defined by

$$
\begin{aligned}
h & =\frac{2[1+(k \sin (k \Pi R \tau(\sigma-1))-\cos (k \Pi R \tau(\sigma-1))) \exp (-\Pi R \tau(\sigma-1))]}{C\left(1+k^{2}\right)} \\
& =\frac{1+[k \sin (k \Pi R \tau(\sigma-1))-\cos (k \Pi R \tau(\sigma-1))] \exp (-\Pi R \tau(\sigma-1))}{\left(1+k^{2}\right)[1-\exp (-\Pi R \tau(\sigma-1))]}(43)
\end{aligned}
$$

Like in Fujita et al. (1999), we focus on the case of a very large economy, that is one with a large $R$. This makes the relationship between the amplification factor $s$ and the frequency $k$ becomes much simpler to handle. We now use the fact that

$$
h \rightarrow \frac{1}{1+k^{2}} \text { as } R \rightarrow \infty
$$

Substituting (37) and (38) into (39), we get $w_{0}=w_{0}\left(l_{0}\right)$. Next $\theta_{0}\left(l_{0}\right)$ and $\omega_{0}\left(l_{0}\right)$ are obtained by using (38) and (40) respectively. By substituting $\omega_{0}\left(l_{0}\right)$ in $(42)$, and then $v_{0}\left(l_{0}\right)$ in $(41)$, we get

$$
\left[s-\frac{1-h}{h}\left(\frac{\mu}{\sigma-1} h+\frac{\mu h^{3}-\left(1+\mu^{2}\right) h^{2}+\mu h}{-(\sigma-1) h^{2}-\mu h+\sigma}-\Delta\right)\right] l_{0}=0
$$


Ignoring the trivial solution $y_{0}=\theta_{0}=w_{0}=\omega_{0}=l_{0}=v_{0}=0$, it follows that a solution to (37), (38), (39), (40), (41), and (42) only exists if the coefficient of $l_{0}$ in (44) is zero, that is, when

$$
s(h)=\frac{1-h}{h}\left[\frac{\mu}{\sigma-1} h+\frac{\mu h^{3}-\left(1+\mu^{2}\right) h^{2}+\mu h}{-(\sigma-1) h^{2}-\mu h+\sigma}-\Delta\right]
$$

The above condition gives the possible values for $s$ and $h$. Since equations (35) are linear, we may construct a general solution to (35) by adding the normal modes (which constitute a complete set).

\subsection{Stability Analysis}

The general question is whether starting from a symmetric spatial equilibrium in which manufacturing is equally divided between regions (such as defined by the long-run equilibrium (33)), a movement of a small number of workers from one region to another is about to lead to the emergence of agglomerations. In the case it does so, the symmetric initial situation is said to be unstable against small perturbations.

Definition 4 A spatial mode defined by its spatial scale $h$ is unstable if its amplificator factor $s(h)$ is strictly positive.

As our goal is to describe whether spatial modes grow or decline in amplitude over time, we will describe the evolution of a spatial mode by its evolution over time. While a positive value of the amplification factor means that the spatial mode will grow in amplitude over time, a negative value of the amplification factor means that the mode is decreasing in amplitude over time. The interpretation is the following. Imagine periodic spatial shocks affecting the long-run equilibrium defined by (33). While a spatial shock $h$ will fade away if $s(h)<0$, it will grow over time if $s(h)>0$.

Definition 5 The long-run equilibrium (33) is unstable if there exists a spatial mode $h \in] 0,1]$ such that $s(h)>0$.

It then means that small initial differences among locations will snowball into larger differences over time, so that the symmetry between identical locations spontaneously breaks. In what follows, we need to focus our analysis on the function $s(h)$ and determine the conditions under which it is positive. 


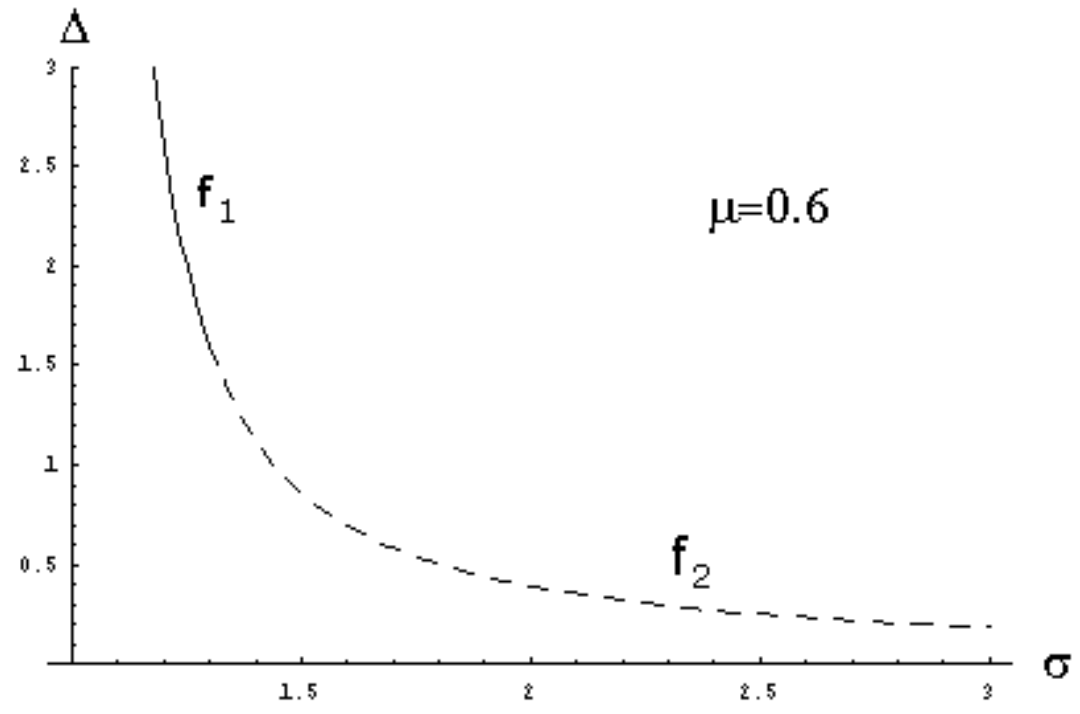

Figure 4: Stability Curve $(\mu=0.6)$

Proposition 1 If the mobility index $\Delta$ is below a critical value $\Delta_{c}$ defined by

$$
\Delta_{c}=\left\{\begin{array}{l}
f_{1}(\sigma, \mu)=\mu\left(1-\sigma^{-1}\right)^{-1}-1, \text { if } \sigma-1 \leq \mu / 2 \\
f_{2}(\sigma, \mu)=\mu^{2}(2 \sigma-1)^{2} /\left[(\sigma-1)\left(\mu^{2}+4 \sigma(\sigma-1)\right)\right], \text { if } \sigma-1>\mu / 2
\end{array}\right.
$$

then the uniform steady state (33) is unstable.

Proof. See Appendix 4.

The impact of migration on the regional structure is twofold. On the one hand, the heterogeneity in location taste leads to the dispersion of workers' across locations, and therefore fosters convergence. On the other hand, the identity of taste for higher utility levels leads to agglomerations of workers in some locations, and therefore contributes to divergence because of increasing returns as in Krugman (1996) or Fujita et al. (1999). The critical value $\Delta_{c}$ in Proposition 1 corresponds to the case where both effects balance exactly. In the parameter space $(\Delta, \sigma, \mu)$, the curve defined by $\Delta=\Delta_{c}$ corresponds to the stability curve, that is the curve along which the amplification factor $s=0$. See Figure 4 for $\mu=0.6$.

When dispersion is the dominant spatial force $\left(\Delta>\Delta_{c}\right)$, the long-run equilibrium (33) is stable, and spatial convergence occurs, meaning that an 
initial spatial shock is absorbed over time. In the particular case of heterogeneity in taste only, meaning in the absence of incentives on behalf of workers to move to higher indirect utility locations, the evolution equation for labor reduces to a pure diffusion equation, in which case regional convergence trivially occurs.

When the drift towards higher utility levels is the dominant spatial force $\left(\Delta<\Delta_{c}\right)$, the long-run equilibrium (33) is unstable, meaning that an initial spatial shock grows in amplitude over time. In this case, the interaction between economies of scale and endogenous market size lead to a cumulative process of agglomeration, see Fujita et al. (1999). As mentioned earlier, the evolution of an arbitrary shock is obtained by an appropriate linear combination of the evolution of the spatial modes.

Proposition 1 is an extension of Krugman (1996) and Fujita et al. (1999) to the case where workers have heterogeneous location tastes. The first part of the stability curve $f_{1}(\sigma, \mu)$ is to be related to Krugman's (1991) stability condition. It says that if the proportion of the manufacturing population $\mu$ is relatively high or if the taste for variety is large ( $\sigma$ is relatively low) compared to $\Delta$, agglomerations emerge. Here in contrast to Krugman (1996), heterogeneity in location taste makes convergence possible. While only high frequency spatial modes are damped in Krugman's work, all spatial modes are damped over time in our model provided that the heterogeneity in location taste is sufficiently higher than the identity of taste for higher utility levels $\left(\Delta>\Delta_{c}\right)$.

Consider the special case of no heterogeneity in location taste $(\Delta=0)$. In this case agglomerations always emerge. First, our result is consistent with Krugman $(1993,1996)$ 's works where migration driven by utility differentials leads to instability. Furthermore, our model differs from Fujita et al. (1999)'s model in one respect only: the range of allowed migration. While migration is supposed to be local here, it has been considered as global in Fujita et al. (1999). Eventhough the spatial adjustments differ in these two models, both spatial economies always diverge. This means that scale economies at the individual firm level and free mobility (either local as here or global in Fujita et al. (1999) or Krugman (1996)) of workers contribute to spatial divergence, regardless of the spatial foresight ability of workers. We also note that unlike the corresponding two-region model of Krugman (1991), divergence always occurs in spatial economies (e.g., Krugman (1996), Fujita et al. (1999), and here). In other words, iceberg transport costs can never balance the agglomeration force in a continuous spatial setting. This is because when 
space is continuous there is always a location toward which transporting the manufacturing good can be made as cheap as you want.

So far, we have found the conditions under which agglomerations may occur. We now turn to the characterization of the instability when it occurs.

Definition 6 The critical spatial mode $h_{c r}$ characterizing the emerging agglomeration is the spatial mode for which $s\left(h_{c r}\right)=0$.

Defined as such, $h_{c r}$ is the spatial scale of the emerging agglomeration, and therefore constitutes a measure of the size of agglomerations.

Proposition 2 The size of agglomerations, when they occur, increases with the taste for variety (inversely related to $\sigma$ ) and the proportion of the manufacturing population $\mu$ and decreases with transport costs.

Proof. Two cases must be analyzed according to $(\sigma-1) \lessgtr \mu / 2$.

First, suppose $(\sigma-1)>\mu / 2$. When instability starts, the shape of the corresponding unstable spatial mode is given by $h_{c r}$ for which $\partial s\left(h_{c r}\right) / \partial h=0$, where $\Delta=f_{2}(\sigma, \mu)$. According to Appendix $4, h_{c r}$ belongs to $] 0,1[$, and we get $h_{c r}=\mu(2 \sigma-1) /\left(\mu^{2}+2(\sigma-1)\right)$. We then have $\partial h_{c r} / \partial \mu=(2 \sigma-1)\left(3 \mu^{2}+\right.$ $2 \sigma-2) /\left(\mu^{2}+2 \sigma-2\right)^{2}>0$ since $(\sigma-1)>\mu / 2$. Therefore, the larger the proportion $\mu$ of the manufacturing population, the larger the size $h_{c r}$ of agglomerations. Also $\partial h_{c r} / \partial \sigma=2 \mu\left(\mu^{2}-1\right) /\left(\mu^{2}+2 \sigma-2\right)^{2}<0$ since $0<\mu<1$. Therefore, the higher the taste for variety (the lower $\sigma$ ), the larger the size $h_{c r}$ of agglomerations. Second, when $(\sigma-1)<\mu / 2$, we look for $h_{c r}$, for which $\partial s\left(h_{c r}\right) / \partial h=0$ and where $\Delta=f_{1}(\sigma, \mu)$. In accordance with Appendix $4, h_{c r}=1$. Finally, since $h$ is always positively related to transport costs (see how $h$ has been rescaled from $k$ in (43)), the higher the transport costs, the smaller the agglomerations.

Proposition 2 makes sense. If the taste for variety is high ( $\sigma$ low), then increasing returns realized at equilibrium are high, and the spatial economy displays a small number of large agglomerations (the corresponding spatial scale $h_{c r}$ being large). Moreover, the effect of a high share of industry in the economy, $\mu$, is to increase the critical spatial mode $h_{c r}$, meaning that starting from a uniform long-run equilibrium, there are a few large agglomerations rather than many small ones. This is because then there are less peasants to supply in the unagglomerated areas. Furthermore, when transport costs are high, it is then sensible for firms to locate closer to local markets in order 
to avoid prohibitive transport costs when supplying immobile peasants in the unagglomerated areas. We finally note that the uniform spatial mode of which the spatial scale is infinite - is the first to become unstable when $(\sigma-1)<\mu / 2$. In this case $\left(h_{c r}=1\right)$, space plays no role in the dimension of the emerging agglomeration.

\section{Concluding comments}

This model sheds light on the distribution of economic agents over space. We have studied the interaction of global trade with labor local migration by decoupling consumption and migration decisions. This has allowed us to stress that local access to information, or risk related to migration, makes the migration decisions quite distinct from consumption decisions. While prices on markets form instantaneously as in the Walrasian tradition, migration is a local process and can be assimilated to a Marshallian adjustment. An evolution equation for labor over space and time has been established.

While two-country models address the question of whether agglomerations take place, continuous spatial models allow to address that of how many agglomerations form, see Fujita et al. (1999). Much of the intuition from the core-periphery model of Krugman (1991) still holds here: the same factors that work toward concentration of economic activity in that model tend to produce fewer, larger agglomerations in our continuous spatial model.

The impact of migration on the regional structure is twofold. First, when driven by utility differentials, it contributes to agglomeration because of the presence of increasing returns resulting from the taste for variety. Second, when reflecting heterogeneous individual choices, it fosters regional convergence. The relative size of both effects is captured by a mobility index. On the one hand, when the mobility index is above the critical value, i.e. when heterogeneity in location taste is high, convergence to the long-run equilibrium is obtained. On the other hand, when the mobility index is below the critical value, agglomerations occur, and their size increases with the taste for variety (inversely related to $\sigma$ ) and the proportion of the manufacturing population, and decreases with transport costs.

The dynamics of the system depends heavily on the nature of economic processes as shown above. It also largely depends on the interaction of the system with the rest of the world. This interaction should be modelled by appropriate boundary conditions. It would necessarily affect the conditions 
under which agglomerations may occur.

\section{References}

[1] Anderson, S., De Palma, A. and J. Thisse (1992), Discrete Choice Theory of Product Differentiation, MIT Press.

[2] Christaller, W. (1966), Central Places in Southern Germany, PrenticeHall, translation from the German, Die Zentralen Orte in Süddeutschland.

[3] Drazin A. and W. Reid (1991), Hydrodynamic Stability, Cambridge University Press.

[4] Fujita M., Krugman, P. and T. Venables (1999), The Spatial Economy: Cities, Regions, and International Trade, Chapter 6: Many Regions and Continuous Space, MIT Press.

[5] Greenberg, J. (1983), Local Public Goods with Mobility: Existence and Optimality of a General Equilibrium, Journal of Economic Theory, 30:1.

[6] Greenwood, M. (1985), Human Migration: Theory, Models, and Empirical Studies, Journal of Regional Science, 25:4.

[7] Isard, W. (1999), Further thoughts on future directions for regional science, The Annals of Regional Science.

[8] Krugman, P. (1991), Increasing Returns and Economic Geography, Journal of Political Economy.

[9] Krugman, P. (1993), On the Number and Location of Cities, European Economic Review, 37, 293-298.

[10] Krugman, P. (1996), The Self-Organizing Economy, Appendix: a Central Place Model, Blackwell Press.

[11] Krugman, P. and A. Venables (1995), The Seamless World: a Spatial Model of International Specialization, NBER working paper $\mathrm{n}^{\circ} 5220$. 
[12] Lösch, A. (1954), The Economics of Location, Yale University Press, translation from the German, Die Räumliche Ord nung der Wirtschaft (1940, 1944).

[13] Mansoorian, A. and G. Myers (1997), On the Consequences of Government Objectives for Economies with Mobile Populations, Journal of Public Economics, 63.

[14] Murray, J. (1993), Mathematical Biology, Springer-Verlag.

[15] Papageorgiou, Y. and T. Smith (1983), Agglomeration as Local Instability of Spatially Uniform Steady-States, Econometrica, 51, 1104-1119.

[16] Shaw, R. (1975), Migration Theory and Fact, Regional Science Research Institute.

[17] Sonnenschein, H. (1981), Price Dynamics and the Disappearance of Short Run Profits: An Example, Journal of Mathematical Economics, 8, 201-204.

[18] Sonnenschein, H. (1982), Price Dynamics Based on the Adjustment of Firms, American Economic Review, 72, 1088-1096.

\section{Appendix 1 Labor Probability Law}

Here we provide a proof for equation (17) when $V^{\prime}$ is not constant.

At time $t$, a worker at location $x$, may have come from a lower indirect utility region or he may come from left or right, or simply may have chosen to stay put. It then follows that the probability $q(x, t)$, at time $t$, for a worker to be at a location between $x$ and $x+d x$ is given by

$$
\begin{aligned}
q(x, t) d x= & \gamma\left[\frac{1}{3} q(x-d \lambda, t-d t)+\frac{1}{3} q(x+d \lambda, t-d t)+\frac{1}{3} q(x, t-d t)\right] d x \\
& +(1-\gamma) F\left(x-V^{\prime} d t, t-d t\right)
\end{aligned}
$$

where $F\left(x-V^{\prime} d t, t-d t\right)$ is the probability that at time $t-d t$, the worker was located between $x-V^{\prime} d t$, and $x+d x-V^{\prime} d t$; see Figure 5 . It is given by

$$
\begin{aligned}
F\left(x-V^{\prime} d t, t-d t\right)= & q\left(x-V^{\prime} d t, t-d t\right)\left[(x+d x)-V^{\prime} d t-\left(x-V^{\prime} d t\right)\right] \\
= & q\left(x-V^{\prime}\left(x-V^{\prime} d t, t-d t\right) d t, t-d t\right) \\
& {\left[x+d x-V^{\prime}\left(x+d x-V^{\prime} d t, t-d t\right) d t\right.} \\
& \left.-\left(x-V^{\prime}\left(x-V^{\prime} d t, t-d t\right) d t\right)\right]
\end{aligned}
$$



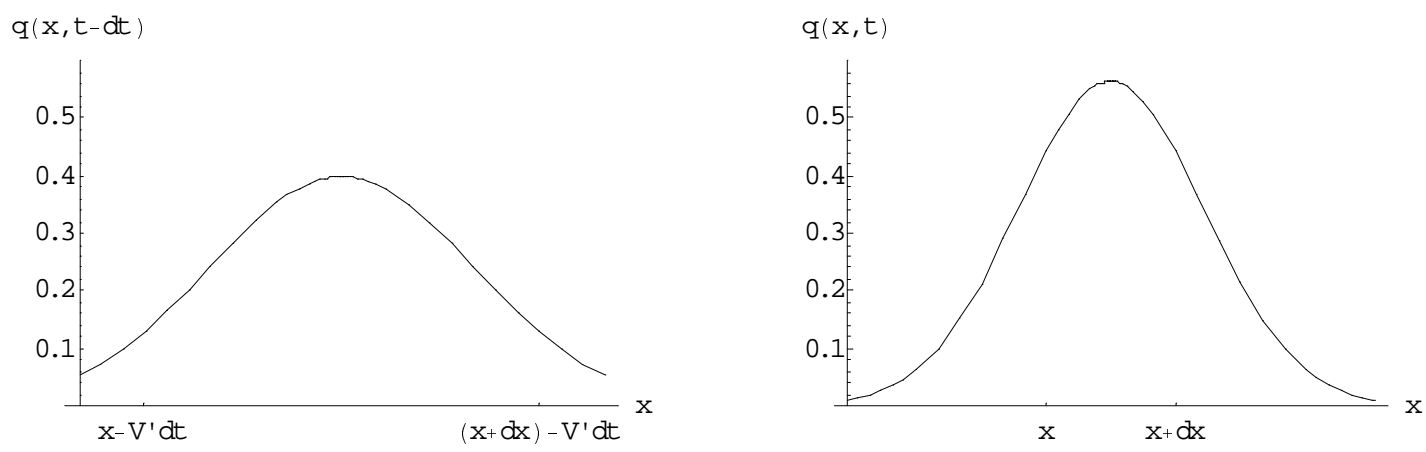

Figure 5: Labor Probability Law

Expanding the RHS of equations (47) and (48) in their Taylor form,

$$
\begin{aligned}
q(x-d \lambda, t-d t)= & q(x, t)-d \lambda \frac{\partial q}{\partial x}-d t \frac{\partial q}{\partial t}+\frac{1}{2}(d \lambda)^{2} \frac{\partial^{2} q}{\partial x^{2}} \\
& +\frac{1}{2} d t^{2} \frac{\partial^{2} q}{\partial t^{2}}+\frac{1}{2} d \lambda d t \frac{\partial^{2} q}{\partial x \partial t}+\ldots \\
q(x+d \lambda, t-d t)= & q(x, t)+d \lambda \frac{\partial q}{\partial x}-d t \frac{\partial q}{\partial t}+\frac{1}{2} d \lambda^{2} \frac{\partial^{2} q}{\partial x^{2}} \\
& +\frac{1}{2} d t^{2} \frac{\partial^{2} q}{\partial t^{2}}-\frac{1}{2} d \lambda d t \frac{\partial^{2} q}{\partial x \partial t}+\ldots \\
q\left(x-V^{\prime} d t, t-d t\right)= & q(x, t)-V^{\prime} d t \frac{\partial q}{\partial x}-d t \frac{\partial q}{\partial t}+\frac{1}{2}\left(V^{\prime} d t\right)^{2} \frac{\partial^{2} q}{\partial x^{2}} \\
& +\frac{1}{2} d t^{2} \frac{\partial^{2} q}{\partial t^{2}}+\frac{1}{2} V^{\prime} d t^{2} \frac{\partial^{2} q}{\partial x \partial t}+\ldots \\
q(x, t-d t)= & q(x, t)-d t \frac{\partial q}{\partial t}+\frac{1}{2} d t^{2} \frac{\partial^{2} q}{\partial t^{2}}+\ldots \\
V^{\prime}\left(x+d x-V^{\prime} d t, t-d t\right)= & V^{\prime}\left(x-V^{\prime} d t, t-d t\right) \\
& -\frac{\partial V^{\prime}}{\partial x}\left(x-V^{\prime} d t, t-d t\right) d x+\ldots
\end{aligned}
$$

Substituting (49) in (47) and (48), while assuming $d \lambda^{2} / d t$ remains finite, yields the probability law (17) governing the evolution of $q(x, t)$ where $V^{\prime}$ is a function of space and time.

\section{Appendix 2 Change of Variables}


Let us define $L^{*}, Y^{*}, \Theta^{*}, W^{*}, \Omega^{*}, V^{*}, x^{*}, t^{*}$ and $\Delta$ such that

$$
\begin{aligned}
(1-\mu) Y^{*} & =A p^{A} ; \mu Y^{*}=L^{*} W^{*} ; V^{*}=m \Omega^{*} \tau(\sigma-1) \\
\Theta^{*} & =\frac{\beta \sigma}{\sigma-1}(\alpha \sigma)^{1 /(\sigma-1)} L^{*^{-1 /(\sigma-1)}} W^{*}\left(\frac{1}{C \tau(\sigma-1)}\right)^{-\frac{1}{\sigma-1}} ; \\
W^{*} & =\frac{\sigma-1}{\beta \sigma}\left(\frac{\mu \beta}{\alpha(\sigma-1)}\right)^{1 / \sigma} Y^{*^{1 / \sigma}} \Theta^{* \frac{\sigma-1}{\sigma}}\left(\frac{1}{C \tau(\sigma-1)}\right)^{\frac{1}{\sigma}} ; \\
\Omega^{*} & =\mu^{\mu}(1-\mu)^{1-\mu} p^{A^{-(1-\mu)}} \Theta^{*^{-\mu}} W^{*} ; \\
x^{*} & =\frac{1}{\tau(\sigma-1)} ; t^{*}=\frac{m \Omega^{*}}{\tau^{2}(\sigma-1)^{2}} ; \Delta=\frac{d}{m \Omega^{*}}
\end{aligned}
$$

where $C$ is defined by $2[1-\exp (-\Pi R \tau(\sigma-1))]$.

By making the substitution $L(x, t) \leftarrow L(x, t) / L^{*} ; Y(x, t) \leftarrow Y(x, t) / Y^{*}$; $W(x, t) \leftarrow W(x, t) / W^{*} ; \Theta(x, t) \leftarrow \Theta(x, t) / \Theta^{*} ; \Omega(x, t) \leftarrow \Omega(x, t) / \Omega^{*} ; V(x, t) \leftarrow$ $V(x, t) / V^{*} ; x \leftarrow x / x^{*} ; t \leftarrow t / t^{*}$ in $(9),(10),(12),(13),(18)$ and (19), we get (27), (28), (29), (30), (31), and (32).

\section{Appendix 3 Perturbation Linearized Equations}

Here we establish the perturbation linearized equations (35). Substituting the decomposition (34) in the evolution equations (9), (10), (12), (13), (18) and (19), yields the following perturbation equations

$$
\begin{aligned}
y(x, t) & =\mu[w(x, t)+l(x, t)+l(x, t) w(x, t)] \\
\theta(x, t)+1 & =\left\{\frac{1}{C} \int_{(x-\Pi R) \tau(\sigma-1)}^{(x+\Pi R) \tau(\sigma-1)}(1+l(z, t))(1+w(z, t))^{1-\sigma} \exp [-|x-z|) d z\right\}^{\frac{1}{1-\sigma}} \\
w(x, t)+1 & =\left\{\frac{1}{C} \int_{(x-\Pi R) \tau(\sigma-1)}^{(x+\Pi R) \tau(\sigma-1)}(1+y(z, t))(1+\theta(z, t))^{\sigma-1} \exp [-|x-z|] d z\right\}^{\frac{1}{\sigma}} \\
\omega(x, t)+1 & =(w(x, t)+1)(\theta(x, t)+1)^{-\mu} \\
\Delta \frac{\partial^{2} l}{\partial x^{2}}(x, t) & =\frac{\partial l}{\partial t}(x, t)+\frac{\partial}{\partial x}(v(x, t)(1+l(x, t))) \\
v(x, t) & =\frac{\partial \omega}{\partial x}(x, t)
\end{aligned}
$$

Then by neglecting second-order terms (i.e. developing $(1+r)^{c} \simeq 1+c r$ ) we get the perturbation linearized equations (35).

$$
y(x, t)=\mu(l(x, t)+w(x, t))
$$




$$
\begin{aligned}
\theta(x, t) & =\frac{1}{C} \int_{(x-\Pi R) \tau(\sigma-1)}^{(x+\Pi R) \tau(\sigma-1)}\left(-\frac{1}{(\sigma-1)} l(z, t)+w(z, t)\right) \exp [-|x-z|] d z \\
w(x, t) & =\frac{1}{\sigma C} \int_{(x-\Pi R) \tau(\sigma-1)}^{(x+\Pi R) \tau(\sigma-1)}(y(z, t)+(\sigma-1) \theta(z, t)) \exp [-|x-z|] d z \\
\omega(x, t) & =w(x, t)-\mu \theta(x, t) \\
\frac{\partial l}{\partial t}(x, t)+\frac{\partial v}{\partial x}(x, t) & =\Delta \frac{\partial^{2} l}{\partial x^{2}}(x, t) \\
v(x, t) & =\frac{\partial \omega}{\partial x}(x, t)
\end{aligned}
$$

\section{Appendix 4 Proof of Proposition 1}

The proof of Proposition 1 draws on the following lemma.

Lemma 1 Consider the degree 2 function $f(x)=a x^{2}+b x+c$. Suppose $b>0$ and $c<0$. Then $f(x)$ admits two distinct real roots in the interval ]0,1[ if and only if

$$
b^{2}-4 a c>0, a<0, a+b+c<0,2 a+b<0
$$

Proof. Sufficiency. Since $b^{2}-4 a c>0$, two real roots exist. Their sum and their product are sure to be positive because $a<0$. Since $a+b+c<0$ and $2 a+b<0$, the largest of the two roots is inferior to 1 .

Necessity. Since $f(x)$ admits two distinct real roots, $b^{2}-4 a c>0$. As they lie in the interval ]0,1[, their sum and product are positive, ie. $a<0$. Moreover, since both roots are inferior to 1 , we have $a+b+c<0$ and $2 a+b<0$.

In what follows, we determine the values of the parameters $(\Delta, \sigma, \mu)$ for which the long-run equilibrium (33) is unstable. This defines the instability region $\Sigma$ in the parameter space $(\Delta, \sigma, \mu)$. To do so, we equivalently determine when $s(h)$ as given by $(45)$, is positive.

Write (45) as

$$
s(h)=\frac{1-h}{h} \frac{\left[a h^{2}+b h+c\right]}{(\sigma-1)\left[-(\sigma-1) h^{2}-\mu h+\sigma\right]}
$$

where 


$$
\begin{aligned}
a & =1+\Delta-\sigma-2 \Delta \sigma-\mu^{2} \sigma+\Delta \sigma^{2} \\
b & =\mu(-1-\Delta+2 \sigma+\Delta \sigma), c=\Delta(1-\sigma) \sigma
\end{aligned}
$$

From (52), we see that $s(h)$ is continuous on $] 0,1]$, admits at most two real roots in ]0, 1[, and $\lim _{h \rightarrow 0} s(h)=-\infty<0$. Hence we may consider two cases.

If $\mu-(1-\mu)(\sigma-1)-\Delta(\sigma-1)<0$, then $\lim _{h \rightarrow 1} s(h)=0^{+}$and the function $s(h)$ takes positive values in ]0,1[ according to the intermediary values theorem. This defines the first instability region $\Sigma_{1}=\{\Delta: 0<\Delta<$ $\left.f_{1}(\sigma, \mu)\right\}$ where $f_{1}(\sigma, \mu)$ has been defined by $\mu\left(1-\sigma^{-1}\right)^{-1}-1$.

If $\mu-(1-\mu)(\sigma-1)-\Delta(\sigma-1)>0$, then $\lim _{h \rightarrow 1} s(h)=0^{-}$. Therefore $s(h)$ will take positive values on $] 0,1]$, iff it has two real roots in between ]0,1[. By using Lemma 1, we show that it is the case when $\sigma-1>\mu / 2$ and $\mu\left(1-\sigma^{-1}\right)^{-1}-1<\Delta<\mu^{2}(2 \sigma-1)^{2} /\left[(\sigma-1)\left(\mu^{2}+4 \sigma(\sigma-1)\right)\right]$. By the substitution of (53) in (51), we obtain the conditions under which $s(h)$ has two real roots in $] 0,1[$,

$$
\begin{aligned}
\Delta & >\frac{1}{\sigma-1} \text { or } \Delta<g_{1}(\sigma, \mu), \\
\Delta & <g_{2}(\sigma, \mu), \Delta>g_{3}(\sigma, \mu), \Delta<g_{4}(\sigma, \mu)
\end{aligned}
$$

where we define $g_{1}(\sigma, \mu)=\left[\mu^{2}(2 \sigma-1)^{2}\right] /\left[(\sigma-1)\left(\mu^{2}+4 \sigma(\sigma-1)\right)\right], g_{2}(\sigma, \mu)=$ $\left[\sigma \mu^{2}+(\sigma-1)\right] /(\sigma-1)^{2}, g_{3}(\sigma, \mu)=\sigma \mu /(\sigma-1)-1$, and $g_{4}(\sigma, \mu)=[2(\sigma-$ $1)+\mu+2 \mu \sigma(\mu-1)] /\left[2(\sigma-1)^{2}+\mu(\sigma-1)\right]$.

Note that $\Delta>(\sigma-1)^{-1}$ is not compatible with the condition involving $g_{4}$, since $1 /(\sigma-1)-g_{4}(\sigma, \mu)=[2(\mu-1) \mu \sigma] /[(\sigma-1)(\mu+2(\sigma-1))]>0$ whatever $\sigma>1$ and $0<\mu<1$. Also, the condition involving $g_{2}(\sigma, \mu)$ is redundant since $g_{1}(\sigma, \mu)-g_{2}(\sigma, \mu)=-\sigma\left(\mu^{2}+2 \sigma^{2}-2\right)^{2} /\left[(\sigma-1)^{2}\left(\mu^{2}+4 \sigma(\sigma-1)\right)\right]<0$. Condition (54) can then be reduced to $\max \left[g_{3}, 0\right]<\Delta<\min \left[g_{1}, g_{4}\right]$. Hence it suffices to show that $g_{4}<g_{3}<g_{1}$ for $\sigma<1+\mu / 2$, and $g_{3}<g_{1}<$ $g_{4}$, for $\sigma>1+\mu / 2$. This is the case because of the following conditions $g_{1}(\sigma, \mu)-g_{3}(\sigma, \mu)=(1-\mu) \sigma(2 \sigma-\mu-2)^{2} /\left[(\sigma-1)\left(\mu^{2}+4 \sigma(\sigma-1)\right)\right] \geq 0$ whatever $\sigma, \mu, g_{4}(\sigma, \mu)-g_{3}(\sigma, \mu)=[(1-\mu) \sigma(2 \sigma-\mu-2)] /[(\sigma-1)(\mu+2(\sigma-1))] \lessgtr 0$ when $\sigma \lessgtr 1+\mu / 2$, and $g_{4}(\sigma, \mu)-g_{1}(\sigma, \mu)=\left[2(1-\mu)(2+\mu-2 \sigma) \sigma\left(\mu^{2}+2(\sigma-1)\right)\right] /[(\sigma-$ $\left.1)(2 \sigma+\mu-2)\left(\mu^{2}+4 \sigma(\sigma-1)\right)\right] \lessgtr 0$ when $\sigma \lessgtr 1+\mu / 2$. This defines the second instability region $\Sigma_{2}=\left\{\Delta: g_{3}(\sigma, \mu)<\Delta<g_{1}(\sigma, \mu), \sigma-1>\mu / 2, \Delta>0\right\}$. 
Finally, we define the instability region $\Sigma$ as $\Sigma=\Sigma_{1} \cup \Sigma_{2}$. To complete the proof of proposition 1 we rewrite $\Sigma=\Sigma_{3} \cup \Sigma_{4}$ where we define $\Sigma_{3}=$ $\left\{\Delta: \Delta<f_{1}(\sigma, \mu), \sigma-1 \leq \mu / 2\right\}, \Sigma_{4}=\left\{\Delta: \Delta<f_{2}(\sigma, \mu), \sigma-1 \geq \mu / 2\right\}$, and $f_{2}(\sigma, \mu)=g_{1}(\sigma, \mu)$. 